\title{
INTELLIGENT AGENTS FOR NETWORK MANAGEMENT
}

\author{
Christian Frei and Boi Faltings !!! add affiliation at bottom (are with) !!!
}

\section{Introduction}

In packet-switched networks, routing can be solved efficiently by local algorithms. This is not the case in circuit-switched networks such as ATM, where routing has been shown to be NP-complete ([4].[6]). Many potential applications such as multimedia transmissions or videoconferences require a quality of service guarantee which can only be given in circuit-switched networks, so this problem is becoming important in practice.

A communication network is a set of nodes that are interconnected by links to permit the exchange of information. We model it as a network graph (see Figure $1 d$ ). A need to exchange information between two nodes is called a demand. Each demand requires a certain quality of service (QoS) when transferred through the network. The required QoS depends on the type of information to transmit and is constrained using several parameters, such as minimal bandwidth, maximal delay, maximal delay jitter, or maximal cell loss rate. In order to satisfy a demand, we must allocate a route between the two endpoints of the demand satisfying the demand's QoS constraints. A route is a path in the network. Assigning a route to a demand and reserving the resources needed is called establishing a circuit. Given a communication network, the problem is to allocate one route for each incomming demand in the network so that the QoS constraints of all demands are satisfied using the available resources of the network.

We propose here a general framework using abstraction techniques ([1],[3]) based on Blocking Islands. The idea is to abstract the original communication network into a hierarchy of simplified graphs where each node, a blocking island, abstracts a part of the network inside which routing of demands requiring a given amount of bandwidth is possible. Each link of an abstract graph identifies then bottleneck links of the network. This hierarchy can be adapted dynamically in reasonable (polynomial) time to reflect the changes in the network's state, such as allocation or deallocation of demands, and link failures, or even link property alterations, and network topology changes (link and node additions). The management of this hierarchy and the routing of demands can easily be distributed to autonomous and intelligent agents.

\section{$\beta$-blocking islands and blocking island hierarchy}

We define the $\beta$-blocking island ( $\beta$-BI) for a node $x$ as the set of all nodes than can be reached from $x$ using links with at least $\beta$ available bandwidth, given the already established circuits. In Figure $1 c$, $N_{1}$ is the $64 K$-BI for $\{a, b, c\}$. A $\beta$-BI thus abstracts a part of a network where there are routes with $\beta$ available bandwidth between any two nodes. Moreover, any such route is "inside" the $\beta$-BI (i.e.. every link of the route has both endpoints in the $\beta$-BI) since, by definition, any link that has one and only one endpoint in a $\beta$-BI has less than $\beta$ available bandwidth: these links identify bottlenecks in the network.

$\beta$-BIs induce a partitionning of the nodes of the network. We call the graph abstracting the communication network the $\beta$-Blocking Island Graph ( $\beta$-BIG), where the nodes are $\beta$-BIs. Figure $1 c$ shows the $64 K$-BIG of the network graph in $d$. The links in a $\beta$-BIG abstract network links between distinct $\beta$-BIs. The available bandwidth on an abstract link corresponds to the maximum available bandwidth 
of the network links it clusters. For instance, the available bandwidth on $L_{1}$, abstracting $l_{5}$ and $l_{6}$, is $16=\max (4.8,16)$.

The recursive decomposition of BIGs for a set of constant bandwidth requirements $\mathcal{B}$ (ordered decreasingly) is called a Blocking Island Hierarchy (BIH) and constitutes a tree of abstractions. In a BIH (see Figure 1), the first level is the $\beta$-BIG of the network graph, where $\beta$ is the first (biggest) element of $\mathcal{B}$. The next levels are BIGs of the preceding ones. The top layer is the 0-BIG of the network that clusters the whole network into a single 0-BI. Construction and maintenance (such as when allocating new circuits, deallocating existing ones when not needed any more, link failures) of a BIH can be realized by polynomial time incremental algorithms.

\section{Routing}

We know that there is a route with $\beta$ available bandwidth between any two nodes of a $\beta$-BI. Therefore there is at least one route $r$ satisfying a demand $d$ requiring $\beta$ bandwidth if the $\beta$-BI $N$ for one endpoint of $d$ in the BIH also contains its other endpoint. Moreover, $r$ is inside $N$. Whatever routing algorithm is used, search space is reduced if we confine it to the subgraph abstracted by $N$. Even better, the search space of the routing algorithm should be set to the $\beta_{i}$-BI $\left(\beta_{i} \geq \beta\right)$ abstracting both endpoints at the lowest level in the BIH in order to restrict it even more. Besides, this heuristic has two beneficial side effects: the more search space is reduced, the less alternative routes are available, therefore easying the choice of a route; it also achieves a load balancing effect because the lower $N$ is in the BIH, the more available bandwidth are on the links it clusters.

When routing a demand is not only subjected to bandwidth requirement but also to other QoS parameters, such as delay and loss rate, a BIH acts as a first filter: we know if there is a route satisfying the bandwidth requirement and in which part of the network graph to look for it. A route satisfying the other QoS constraints can then be searched in that subgraph. Hereby, as possibly much time is gained through search space reduction, more complex routing algorithms can be used then before or more alternative routes can be examined in order to choose the best one. As many end-to-end QoS parameters are not independent from the available bandwidth resources in the network, e.g., if the load increases, delay, delay jitter and loss increase too, using a bandwidth filter such as a BIH proves to be a very good choice, besides the fact that bandwidth is a critical QoS parameter for any multimedia transmission.

A BIH gives also the means to compare a priori equivalent routes in order to decide for the "best" one, besides the length criterion (the shorter the route, the less resources are used). We know that the higher a link is abstracted in the $\mathrm{BIH}$, the more bandwidth critical it is. Comparison of two routes can then be based on the criticalness of its links, or at which level in the BIH each route is "inside" a BI. Another way to compare routes is to analyze the consequences each route has on the BIH. The best route is the route that causes the less splittings of blocking islands in the BIH: obviously, the more splittings, the more links become critical leading to more allocation failures of demands because no route satisfying the new demands are available. This "forward-checking" like criterion has also a load balancing effect.

The methods described are only applicable when there is a $\beta_{u}$ level in the BIH for a demand requiring $\beta_{u}$. In practice, there are typical bandwidth requirements and they are not that many. A BIH with all corresponding levels is therefore feasable.

Nevertheless, building and maintaining a BIH has a cost. This cost would be prohibitive when routing short-lived information flows, such as electronic mail. A realistic system must therfore use traditional routing algorithms for routing short-lived demands and a BIH to manage long-lived connections (e.g., phone call, video-on-demand). In order to accomodate the short-lived demands, some resources must be 
reserved permanentely on the network's links: from the BIH's point of view, there is a single permanent circuit on each link. How much resources must be reserved for these short-lived connections depend on traffic estimations. These estimations can however be conservative because most short-lived demands do not have stringent QoS constraints.

\section{Distributed resource allocation by intelligent agents}

A key research in communication networks nowadays is how to move management from centralized to distributed, in order to overcome the increasing complexity of this task. Most solutions, such as $P-N N I$ ([2]) or HYBRID ([5]), are based on a hierarchy of autonomous intelligent agents which have local decision-making capabilities, but co-operate to resolve conflicts. Higher level agents arbitrate unresolvable disputes between peer agents. In these systems, an agent reigns over an arbitrarily and statically defined subnetwork, in most cases an administrative domain of some kind. For instance, a university could be managed as follows: an agent is in charge in each lab, whereas other agents each oversee a departement and a single one rules the university. These domains do not reflect the easy routing parts of the network and do not evolve.

The management and maintenance of a BIH is easily distributed to intelligent agents. Each blocking island is ruled by an autonomous agent, a BI leader, which is responsible for resource allocation and management in its domain. Each agent is ruled by one and only one father on the next BIH level. The top level leader is then the highest agent in charge. Our hierarchy of agents yields the abstraction tree of the BIH and can be managed the same way as the hierarchical systems described above, except that a BIH is dynamic, with same performance gain. Our agents are therefore dynamic and have additionnal responsability: the creating and killing of agents when child blocking islands are splitted and merged, respectively.

Circuit establishing is effectively managed by our agents. When a new demand arises, issued by a network node $x$ which needs to communicate with another node $y$ at $\beta$ bits/sec, $x$ asks its direct leader to establish that connection. The leader passes on the demand characteristics to its own leader, until the leader at $\beta$ level is reached. The latter is then responsible for establishing a circuit for the new demand, that is, find a route statisfying its QoS constraints and reserve the required resources among the chosen route. The $\beta$-leader does not have to communicate with peer agents in order to negotiate resources, because any route satisfying the new demand only uses resources he is responsible for. This is the main advantage of our distributed BIH over systems such as P-NNI and HYBRID: our domains reflect current bandwidth resource availability. Routing and resource reservation are hereby purpusefully distributed. As a result, less co-operation and negotion between BI leaders are required than between agents of the other systems when circuits must be established: our agents are more autonomous.

\section{Conclusion}

By distinguishing the "easy" parts from the critical ones in the network, this BIH framework allows a straight answer about whether a route exists satisfying the bandwidth requirement of a demand and the use of existing routing algorithms more efficiently because the problem size is reduced to a blocking island. Network utilization is improved as this decomposition provides to the routing algorithms more knowledge about the network's state than previously available: fewer demands will have to be rejected.

A distributed BIH manages bandwidth resources much more efficiently than other distributed systems, resolving their arbitrary domains weekness, while achieving other tasks (such as network monitoring and performance analysis) just as well at only a slight cost: the management of dynamic agents.

Furthermore, this framework is network technology independent, as far as circuit-switched networks are considered. 
(a)

(b)

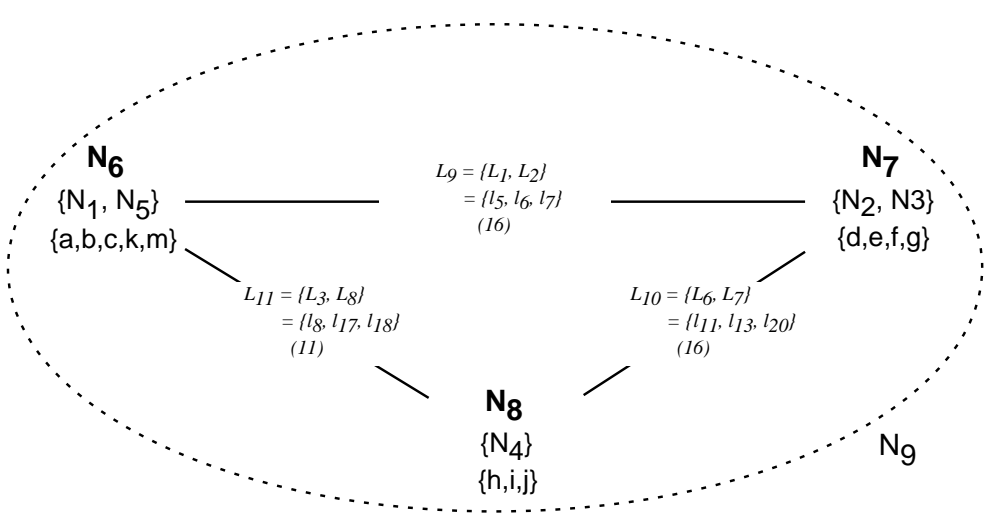

19.2K-BIG

(c)

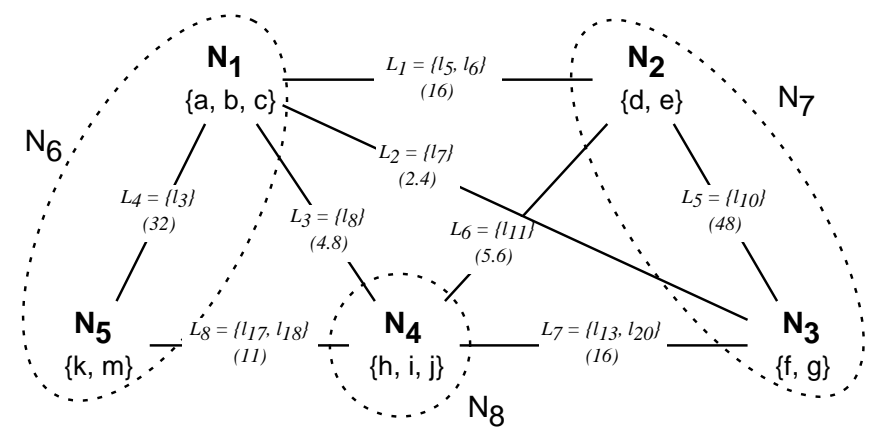

Network

(d)

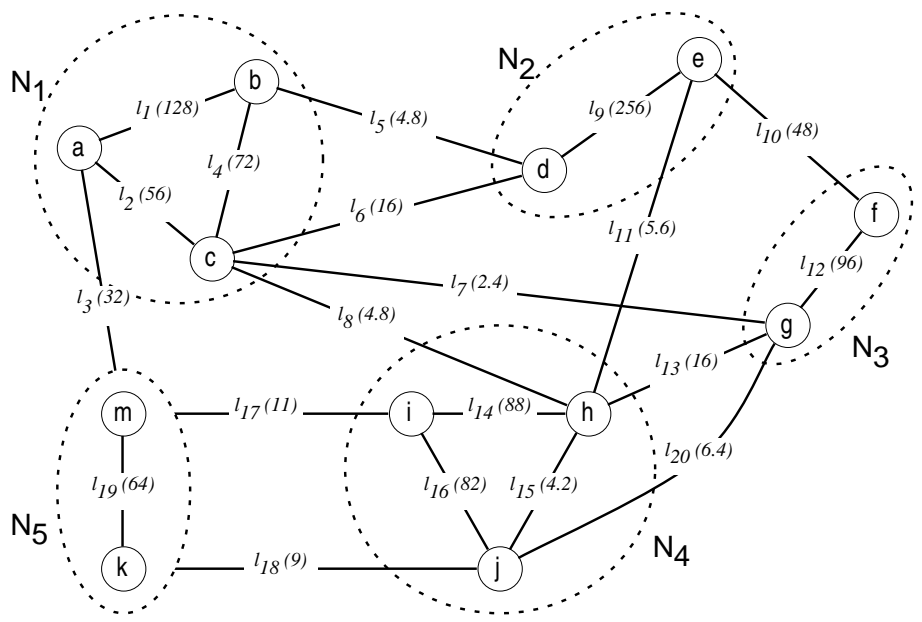

Figure 1: The blocking island hierarchy for bandwidth requirements $\{64 K, 19.2 K\}$. The weights (in brackets) on the links are the available bandwidths. Abstract nodes' and links' description include their children and (if not the same) network children in braces. (a) the 0-BIG. (b) the $19.2 \mathrm{~K}$-BIG. (c) the $64 \mathrm{~K}$-BIG. (d) the network graph. 


\section{References}

[1] Dean Allemang and Beat Liver. How can we communicate with computers? Abstractions: their purpose and application in telecommunications. ComTec: Technische Mitteilungen Swiss Telecom PTT, (10):948 - 956, 1995.

[2] The ATM Forum. P-NNI 1.0 Specification, May 1996.

[3] Berthe Y. Choueiry. Abstraction Methods for Resource Allocation. PhD thesis, Swiss Federal Institute of Technology in Lausanne (EPFL), Switzerland, October 1994. Thesis No 1292.

[4] Michael R. Garey and David S. Johnson. Computers and intractability. A guide to the theory of NP-Completeness. W.H. Freeman and Company, New-York, 1979.

[5] Fergal Somers. HYBRID: Intelligent Agents for Distributed ATM Network Management. In Working notes of the Workshop on Intelligent Agents for Telecom Applications, ECAI'96, Budapest, Hungary, 1996.

[6] Zheng Wang and Jon Crowcroft. Quality-of-Service Routing for Supporting Multimedia Applications. IEEE Journal on Selected Areas in Communications, 14(7):1228-1234, September 1996. 